\title{
Bacterial fecal microbiota is only minimally affected by a standardized weight loss plan in obese cats
}

Moran Tal ${ }^{1,2}$, J. Scott Weese ${ }^{3}$, Diego E. Gomez ${ }^{1}$, Myriam Hesta ${ }^{4}$, Joerg M. Steiner ${ }^{5}$ and Adronie Verbrugghe ${ }^{1 *}$

\begin{abstract}
Background: Research in humans and mice suggests that obesity influences the abundance and diversity of gastrointestinal (Gl) microbiota, and that an "obese microbiome" influences energy metabolism and fat storage in the host. Microbiota membership and composition have been previously assessed in healthy cats. However, research investigating the effects of obesity and weight loss on the cat's fecal microbiota is limited. Therefore, this study's objective was to evaluate differences in fecal microbial abundance and biodiversity, as well as serum cobalamin and folate concentrations in obese cats, before and after weight loss, and compare to lean cats. Fourteen lean and 17 obese healthy client-owned cats were fed a veterinary therapeutic weight loss food at maintenance energy requirement for 4 weeks. At the end of week 4, lean cats finished the study, whereas obese cats continued with a 10-week weight loss period on the same food, fed at individually-tailored weight loss energy requirements. Body weight and body condition score were recorded every 2 weeks throughout the study. At the end of each period, a fecal sample and food-consumption records were obtained from the owners, and serum cobalamin and folate concentrations were analysed. DNA was extracted from fecal samples, polymerase chain reaction (PCR) was performed, and products were sequenced using next-generation sequencing (Illumina MiSeq).

Results: No significant differences in the relative abundance of taxa and in biodiversity indices were observed between cats in either group ( $P>0.05$ for all tests). Nevertheless, some significantly enriched taxa, mainly belonging to Firmicutes, were noted in linear discriminant analysis effect size test in obese cats before weight loss compared to lean cats. Serum cobalamin concentrations were significantly higher in lean compared to obese cats both before and after weight loss. Serum folate concentrations were higher in obese cats before weight loss compared to after.

Conclusions: The association between feline obesity and the fecal bacterial microbiota was demonstrated in enriched taxa in obese cats compared to lean cats, which may be related to enhanced efficiency of energyharvesting. However, in obese cats, the fecal microbial abundance and biodiversity were only minimally affected during the early phase of a standardized weight loss plan.
\end{abstract}

Keywords: Feline obesity, Fecal microbiome weight loss, Microbial diversity, Folate, Cobalamin

\footnotetext{
* Correspondence: averbrug@uoguelph.ca

'Department of Clinical Studies, Ontario Veterinary College, University of Guelph, Guelph, ON N1G 2W1, Canada

Full list of author information is available at the end of the article
}

(C) The Author(s). 2020 Open Access This article is licensed under a Creative Commons Attribution 4.0 International License, which permits use, sharing, adaptation, distribution and reproduction in any medium or format, as long as you give appropriate credit to the original author(s) and the source, provide a link to the Creative Commons licence, and indicate if changes were made. The images or other third party material in this article are included in the article's Creative Commons licence, unless indicated otherwise in a credit line to the material. If material is not included in the article's Creative Commons licence and your intended use is not permitted by statutory regulation or exceeds the permitted use, you will need to obtain permission directly from the copyright holder. To view a copy of this licence, visit http://creativecommons.org/licenses/by/4.0/ The Creative Commons Public Domain Dedication waiver (http://creativecommons.org/publicdomain/zero/1.0/) applies to the data made available in this article, unless otherwise stated in a credit line to the data. 


\section{Background}

Obesity is a prominent problem in both humans and companion animals. There are a few definitions for obesity, but they are all in agreement that the term reflects an excess of body fat that jeopardizes the individual's health [1-4]. Estimations from the last decade indicate that in Europe, 11.5 and $26.8 \%$ of cats are overweight or obese in the UK and France respectively, while $59.5 \%$ of cats were reported as overweight of obese in the USA and $63 \%$ in New Zealand [5-8].

Obesity is mainly caused by an imbalance between energy intake and energy expenditure [9]. Additional factors contribute to obesity, such as genetics, age, sex, neutering, physical inactivity, and endocrine disease [7, $8,10-12]$. However, more recent findings indicate that the gastrointestinal (GI) microbiota (i.e., the gastrointestinal microbial community) plays an important role in the development of obesity. The microbiota can be addressed as a "metabolic organ", acting to support the host with metabolic functions that the host is not capable of performing, such as fermentation of plant polysaccharides [13-15]. The microbiota can also affect metabolic pathways in the host, for example promote metabolic pathways that enhance adipose tissue storage $[13,16]$. Moreover, the microbiota is also involved in low-grade inflammation, which occurs with obesity. It is suggested that lipopolysaccharide (LPS), a structural component of the external membrane of gram-negative bacteria, triggers inflammation in the host through the innate immune response [17].

Increased relative abundance of Firmicutes versus Bacteroidetes, and hence a higher Firmicutes-to-Bacteroidetes ratio were observed in obese humans and mice compared to their lean counterparts $[18,19]$. However, some studies did not observe these differences [15]. Obesity was also associated with reduced microbial richness and diversity in humans and mice [20, 21]. Nonetheless, little research exists in companion animals to demonstrate similar findings. Research in dogs demonstrated increased relative abundance of the phylum Actinobacteria and genus Roseburia in lean versus obese dogs [22]. However, the subjects differed in their environment (i.e., lean research dogs and obese pet dogs), and the dogs were not fed the same food [22]. Limited research on the relationship between obesity and the fecal microbiota has also been performed in cats. One study examined the differences of the microbiota between lean and overweight/obese cats using two fecal collection methods - rectal swabs versus litter box samples. Microbiota differences were found between lean and obese cats with both collection methods. However, shelter cats were used, with the medical history being unknown, and various different diets being consumed (i.e., no diet adaptation period). Hence, the results may have been affected by the diet or an unknown medical condition. Also the effect of weight loss was not assessed [23]. A second feline study examined the effect of weight loss on the microbiota in eight obese kennel cats. However, the sample size was quite small, results were not compared to matching lean kennel cats, and the study environment was well controlled, and thus, less reflective of the true effects in the general cat population [24]. A third feline study, examined the effects of body composition, 6 weeks of energy restriction and neutering on the feline fecal mictobiota of cats. Nonetheless, the weight loss period was quite short, the sample size was small and the cats were specific-pathogen-free kennel cats, so again not reflective of the general pet cat population [25].

Obesity may impact intestinal permeability through deviation from normal microbial structure (i.e., bacterial dysbiosis), which could cause an increase in lipopolysaccharide (LPS)-producing bacteria and overall LPS production [26]. The direct assessment of intestinal permeability via tight junction protein expression, for example, requires harvesting of intestinal tissue [27]. However, measuring serum cobalamin and folate can be used for non-invasive assessment of intestinal health in humans, dogs, and cats [28, 29]. Gastrointestinal bacterial dysbiosis can be a cause of reduced serum concentrations of cobalamin and may alter microbial folate synthesis [28]. Low serum cobalamin and/or folate status have been observed in various obese human populations, for example children and adolescents, post-menopausal women and pre-operative bariatric surgery patients. It is however uncertain if this is due to volumetric dilution of the blood of obese patients, low dietary intake, reduced uptake by the intestinal epithelium, increased catabolism, and sequestration in adipose tissue, or changes in the gut microbiota profiles [30-33]. To the authors' knowledge, serum cobalamin and folate concentrations have not been assessed in feline obesity.

Therefore, the aim of the current study was to investigate the fecal microbiota and serum cobalamin and folate concentrations in obese client-owned cats before and after a 10-week standardized weight loss plan and to compare these to a lean control group, while all cats were acclimatized for 4 weeks to the same diet before study enrollment. It was hypothesized that the feline fecal microbial composition and structure will differ between lean and obese cats and that these microbial differences will revert with weight loss in obese cats, to resemble the fecal microbiota of lean cats. It was also hypothesized that a reduced cobalamin concentration and increased folate concentration will be observed in obese cats before weight loss compared with lean cats and obese cats after weight loss. 


\section{Results}

Fourteen lean and 17 obese cats were enrolled into the study. One obese cat did not complete the study due to reduced owner cooperation with food restriction, which resulted in weight maintenance rather than weight loss. Results from this cat were included for the obese cats before weight loss (OBWL) time point, but not for the obese cats after weight loss (OAWL) time point. Only one stool sample and food intake data were obtained for one cat because of its fractious nature, leaving 13 cats for assessment of body weight $(\mathrm{BW})$ and body composition in the LEAN group. All cats tolerated the food well, no cat refused to eat the food, and none showed signs of illness or maldigestion (mean food consumption \pm standard deviation (SD) within groups - LEAN: $215.2 \pm 35.69 \mathrm{kcal}, \quad$ OBWL: $217.8 \pm 24.33 \mathrm{kcal}$, OAWL: $134.6 \pm 9.20 \mathrm{kcal})$.

\section{Body weight and body composition measurements}

Body weight remained stable in all cats during the 4week adaptation period. However, mean BW was significantly lower in the LEAN versus the OBWL $(P<0.0001)$ group. The 10-week weight loss plan was successful as body weight was significantly lower for OAWL compared to OBWL $(P<0.0001)$ measurements. On average cats lost $0.94 \pm 0.28 \%$ of their initial body weight per week. Also, a significant decrease in body condition score (BCS) between OBWL and OAWL was observed $(P=0.001)$. Body mass index (BMI) and girth in LEAN were significantly lower compared to OBWL $(P<$ 0.0001), and were also significantly lower for OAWL compared to OBWL $(P<0.0001)$. Altogether, BW, BCS, $\mathrm{BMI}$ and girth were still significantly higher in the obese cat group than in LEAN cats after the 10-week weight loss plan $(P<0.0001$ for BW, BCS and girth, and $P<$ 0.0002 for BMI) (Table 1).

\section{Serum Cobalamin and Folate concentrations}

Serum cobalamin concentrations were higher in LEAN cats compared to OBWL cats $(P=0.009)$ and were also higher in the LEAN group compared to the OAWL group $(P=0.021)$. No differences were observed in serum concentrations of cobalamin between OBWL and OAWL measurements. Serum folate concentrations were significantly higher for OBWL compared to OAWL $(P=0.003)$. No other differences were observed for serum folate concentrations between groups or time points (Table 2).

\section{Fecal microbiota analyses}

Fecal analyses resulted in a total of 9,193,399 sequences that passed all filters, with a median of 105,984 sequences per sample (range: 22,314-557,519). A random
Table 1 Body weight and body composition measurements in lean and obese cats before and after weight loss

\begin{tabular}{llll}
\hline & LEAN & OBWL & OAWL \\
& Mean \pm SD & Mean \pm SD & Mean \pm SD \\
\hline BW (kg) & $4.49 \pm 0.22^{a, c}$ & $6.95 \pm 1.32^{a, b}$ & $6.30 \pm 1.13^{b, c}$ \\
BMI (kg/m²) & $41.58 \pm 4.67^{a, c}$ & $60.45 \pm 12.05^{a, b}$ & $55.61 \pm 11.02^{b, c}$ \\
Girth (cm) & $38.38 \pm 3.79^{a, c}$ & $52.12 \pm 4.89^{a, b}$ & $48.25 \pm 5.55^{b, c}$ \\
& Median (Min-Max) & Median (Min-Max) & Median (Min-Max) \\
BCS (1-9/9) & $5(4 \text { to 5) })^{a, c}$ & $9(8 \text { to 9) })^{a, b}$ & $8(6 \text { to 9) })^{b, c}$ \\
\hline
\end{tabular}

$O B W L$ obese before weight loss, OAWL obese after weight loss, $B W$ body weight, $B M I$ body mass index, $B C S$ body condition score, $S D$ standard deviation, Min minimum, Max maximum

The data presented here represent the BW and body composition measurements of healthy lean cats ( $\operatorname{LAN}, n=13$ ) and obese cats following a 4-week adaptation period with a veterinary therapeutic food intended for weight loss and adult maintenance (OBWL, $n=17$ ), and obese cats after a 10week weight loss period on the same food (OAWL, $n=16$ )

${ }^{a}$ Significant difference between LEAN to OBWL $(P<0.0001$ for BW, girth, BMI and $B C S$ ); Student T-test (BW, BMI and girth) or Wilcoxon Mann-Whitney (BCS) ${ }^{\mathrm{b}}$ Significant difference between OBWL to OAWL $(P<0.0001$ for BW, girth and $\mathrm{BMl} ; P=0.001$ for $\mathrm{BCS}$ ); Paired T-test (BW, BMl and girth) or Wilcoxon Signed-Rank (BCS)

${ }^{c}$ Significant difference between LEAN to OAWL $(P<0.0001$ for BW, girth, and $\mathrm{BCS} ; P<0.0002$ for $\mathrm{BMI})$; Student T-test (BW, BMI and girth) or Wilcoxon Mann-Whitney (BCS)

subsample of 22,314 sequences per sample was used for sample normalization. One thousand forty-two operational taxonomic units (OTUs) were formed.

\section{Relative abundance}

Median relative bacterial abundance was examined across all taxa between groups. After performing a Benjamini-Hochberg adjustment, significant differences between groups were no longer identified for any taxa (Figs. 1 \& 2, Table 3, $P>0.05$ ). The most abundant phyla in all groups were (in a descending order) Firmicutes, Proteobacteria, Actinobacteria, and Bacteroidetes. The most abundant genera in all groups were (in a descending order): Clostridium_XI, Megasphaera, Erysipelotrichaceae_incertae_sedis, Lachnospiraceae and blautia.

\section{Alpha and beta diversity indices}

There were no significant differences in alpha diversity matrices between groups or time-points (all $P>0.05$ ) (Fig. 3). There were also no differences in community membership (Classical Jaccard index: unifrac $P=0.17$; parsimony $P>0.05$ for all comparisons) or population structure (Yue \& Clayton: unifrac $P=0.93$; parsimony $P>0.05$ for all comparisons) between groups or timepoints.

No significant clustering per group was evident in a dendogram of the Classical Jaccard index representing the community membership of the fecal microbiota, and there was a strong intra-cat relationship (Fig. 4). Principal coordinate analyses also demonstrated no apparent clustering based on community membership (Fig. 5) or 
Table 2 Serum cobalamin and folate concentrations in lean cats and in obese cats before and after weight loss

\begin{tabular}{llll}
\hline Analytes & LEAN & OBWL & OAWL \\
& Median (Min-Max) & Median (Min-Max) & Median (Min-Max) \\
\hline Cobalamin $(\mathrm{pg} / \mathrm{mL})(350-1499 \mathrm{pg} / \mathrm{mL})^{\mathrm{a}}$ & $913(821-973)^{\mathrm{b}, \mathrm{d}}$ & $882.0(702-928)^{\mathrm{b}}$ & $879.0(571-974)^{\mathrm{d}}$ \\
& Mean (LL-UL) & Mean (LL-UL) & Mean (LL-UL) \\
Folate $(\mathrm{ng} / \mathrm{mL})(9.7-21.6 \mathrm{ng} / \mathrm{mL})^{\mathrm{a}}$ & $18.8(16.2-21.8)$ & $20.1(17.7-22.9)^{c}$ & $17.4(15.3-19.9)^{c}$ \\
\hline
\end{tabular}

OBWL obese before weight loss, OAWL, obese after weight loss, Min minimum, Max maximum, LL-UL lower limit - upper limit

The data presented here represent the cobalamin and folate serum concentrations of healthy lean cats (LEAN, $n=13$ ) and obese cats following a 4-week adaptation period with a veterinary therapeutic food intended for weight loss and adult maintenance (OBWL, $n=17)$, and obese cats after a 10-week weight loss period on the same food (OAWL, $n=16$ )

${ }^{a}$ Normal reference-range provided by the Gastrointestinal Laboratory, Texas A\&M University

${ }^{b}$ Significant difference between LEAN to OBWL (and $P=0.0057$ for cobalamin and $P=0.2989$ for folate); Wilcoxon Mann-Whitney (cobalamin) or Student T-test after log transformation (folate)

'Significant difference between OBWL to OAWL ( $P=0.8209$ for cobalamin and $P=0.0321$ for folate); Wilcoxon Signed Rank (cobalamin) or Paired T-test after log transformation (folate)

${ }^{\mathrm{d}}$ Significant difference between LEAN to OAWL ( $P=0.0149$ for cobalamin and $P=0.6058$ for folate); Wilcoxon Mann-Whitney (cobalamin) or Student T-test after log transformation (folate)

structure between groups (analysis of molecular variance (AMOVA) $P>0.05$ and homogeneity of molecular variance (HOMOVA) $P>0.05$ for all comparisons).

\section{Linear discriminatory analysis effective size (LEfSe)}

LEfSe analysis failed to identify enriched taxa between the OBWL and OAWL time-points. However, a comparison of the LEAN to the OBWL group revealed 13 OTUs that were differentially enriched in the OBWL group (Table 4), six of which are members of the phylum Firmicutes, two that belong to the phylum Proteobacteria and two to the phylum Actinobacteria. One OTU of the phylum Tenericutes was found differentially enriched in the LEAN group. When comparing LEAN to the OAWL cats, the only difference was enrichment of Pseudomonas (phylum Proteobacteria).

\section{Discussion}

Based on human and rodent studies, it was expected that there would be differences of the fecal microbiota between lean and obese cats and that these microbial differences would revert with weight loss in obese cats. However, the bacterial fecal microbiota was only minimally affected in the current study.

One study reported on a $50 \%$ decrease in the relative abundance of Bacteroidetes in genetically-modified

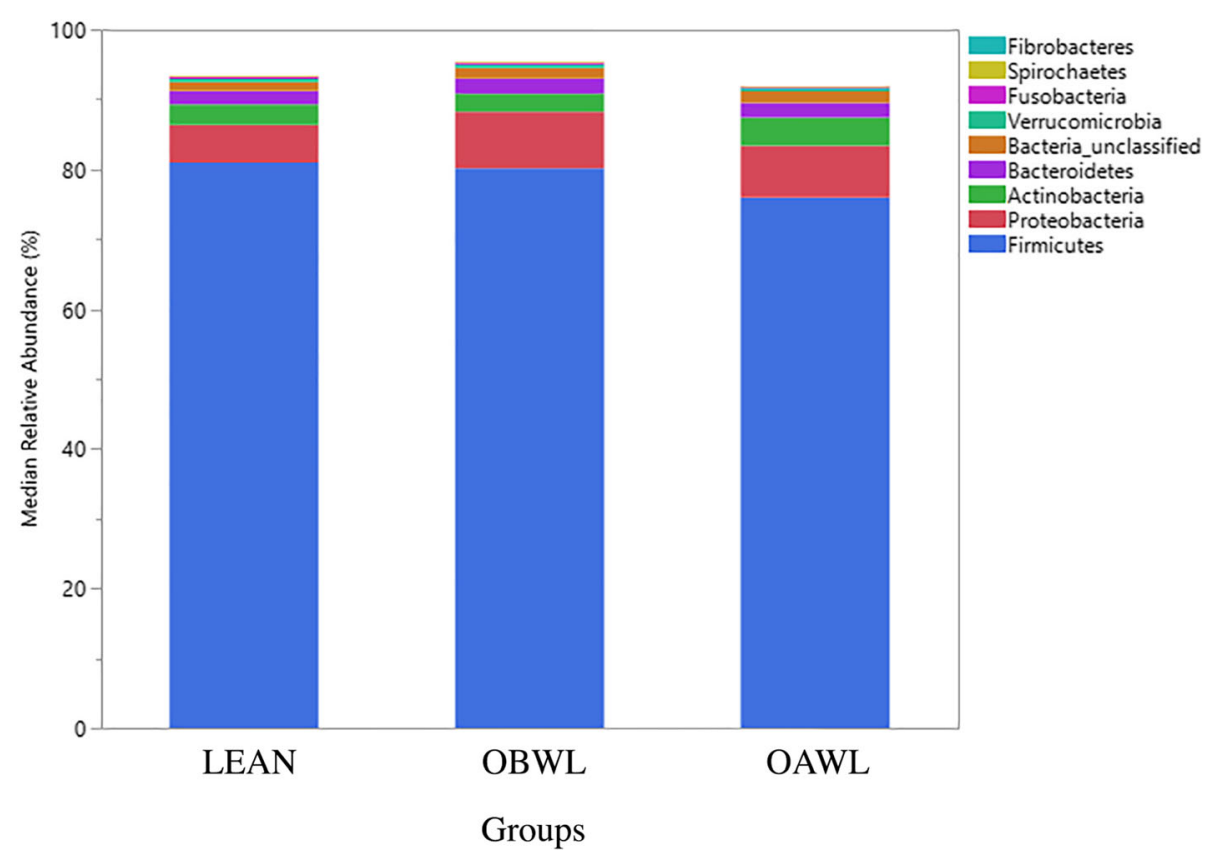

Fig. 1 The relative abundances of predominant phyla originating from fecal samples of healthy lean cats (LEAN, $n=14)$ and obese cats (OBWL, $n=17$ ) following a 4-week adaptation period with a veterinary therapeutic food intended for weight loss and adult maintenance, and obese cats after a 10-week weight loss period on the same food (OAWL, $n=16)$ 


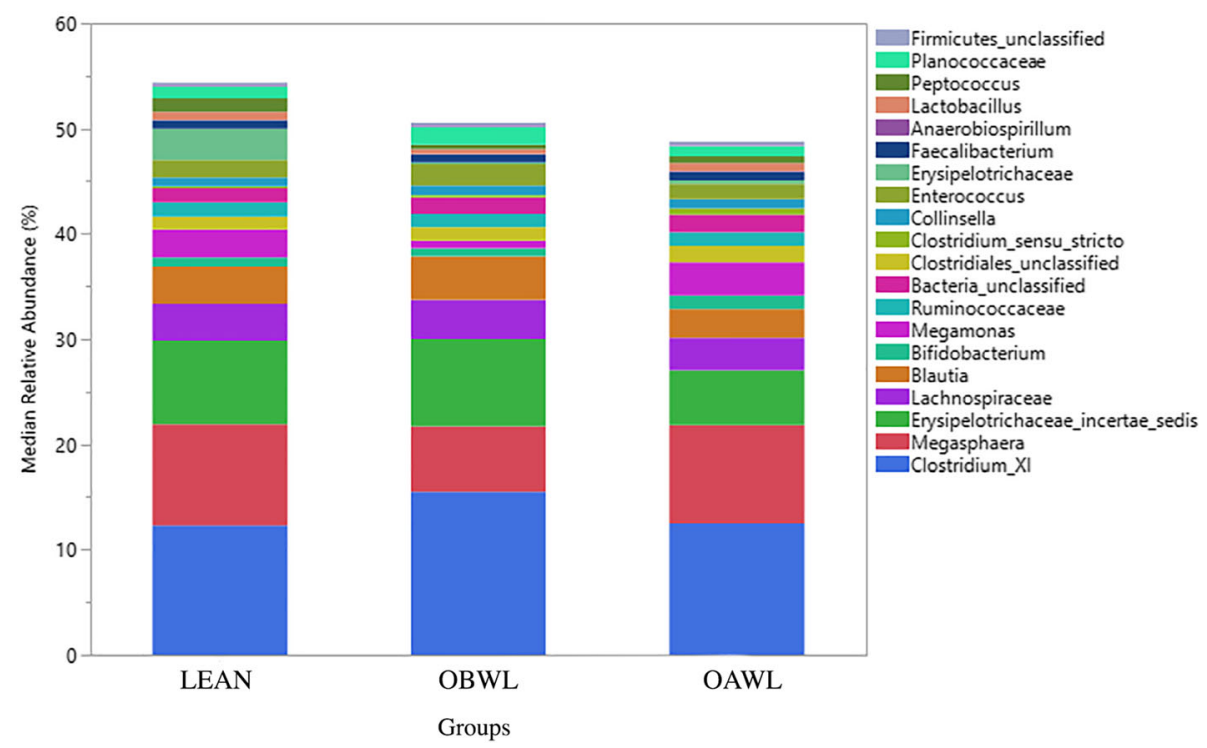

Fig. 2 The relative abundances of predominant genera originating from fecal samples of healthy lean cats (LEAN, $n=14)$ and obese cats (OBWL, $n=17)$ following a 4-week adaptation period with a veterinary therapeutic food intended for weight loss and adult maintenance, and obese cats after a 10-week weight loss period on the same food (OAWL, $n=16$ )

obese mice compared to lean mice that were fed the same high-polysaccharide diet. This change was also associated with a corresponding increase in Firmicutes and reduced diversity [18]. Similar changes were subsequently identified in obese adult humans [19]. In addition, weight loss, induced by using fat and carbohydrate-restricted diets, was found to restore the original ratio between Firmicutes to Bacteroidetes with the restoration being correlated to the percentage of weight loss [19]. More recent findings indicate that diet composition rather than caloric intake, had the greatest effect on the fecal microbiota of mice, when comparing lean mice to obese mice after weight loss (i.e., two groups of mice with similar weight and body composition) [34]. These findings corroborated other studies that showed that dietary effects (especially fat content) and initial body composition during weight reduction of obese mice and humans were the main contributors to a significant change in the fecal microbiota [35-37]. In contrast to those previous findings, such a broad effect was not noted in the current feline study as the relative abundance of taxa and biodiversity indices were not different between lean and obese cats, and in obese cats before and after weight loss. This could relate to various factors, such as differences between cats and other species, environmental influence, the pathophysiology of obesity in cats, the degree and duration of the initial obese status, the nature of the weight loss intervention, degree of cooperation of owners and the duration of the study period. Although in the current study, the weight loss period lasted only 10 weeks and the final fecal sample was taken when weight loss was still occurring, the food used was the same for all groups/periods, excluding diet as a confounder.

Despite the lack of broad taxonomic changes through comparison of relative abundances, differences in the microbiota were identified when comparing lean to obese cats before weight loss. Six of the 13 OTUs that were significantly enriched in obese cats before weight loss belong to Firmicutes, a finding which is consistent with the over-representation of certain Firmicutes members in obese subjects in other species [18]. In humans such an obesity-associated increase in Firmicutes was thought to be related to an increased ability of energy harvest and storage [18]. Additional research in genetically-related obesity in humans, as well as in mice fed a high-fat diet, was not able to correlate microbiota composition and membership changes with energyharvest markers (i.e., short chain fatty acids (SCFA) and energy content in feces). Therefore, it was suggested that the changes observed indicated a potential microbial adaptation to diet or obesity over time, and hence, a more complex, and not completely understood, interaction between the microbiota and energy harvesting [38]. In a recent feline study, the influence of neutering, body composition, and 6 weeks of energy restriction on fecal microbiota of cats, was assessed. All cats were adapted to the study diet for 8 weeks before samples were obtained. No major differences were observed between groups in regards to bacterial composition and structure, however, a significant small difference in the abundance of Firmicutes between the lean neutered 
Table 3 Relative abundances of the fecal microbiota members of healthy lean cats and obese cats before and after weight loss

\begin{tabular}{|c|c|c|c|}
\hline & \multicolumn{3}{|c|}{ Medians (Min-Max) of relative abundance (\%) } \\
\hline & LEAN & OBWL & OAWL \\
\hline \multicolumn{4}{|l|}{ Phyla $(9 / 9)^{a}$} \\
\hline Firmicutes & $81.0(63.7-91.0)$ & $80.2(5.3-93.4)$ & $76.0(46.3-92.1)$ \\
\hline Proteobacteria & $5.4(1.9-23.2)$ & $8.2(2.9-19.0)$ & $7.4(1.2-43.0)$ \\
\hline Actinobacteria & $2.9(0.8-32.8)$ & $2.5(0.4-30.1)$ & $4.0(0.5-13.6)$ \\
\hline Bacteroidetes & $1.9(0.4-5.1)$ & $2.1(0.5-4.3)$ & $2.0(0.6-5.3)$ \\
\hline Bacteria_unclassified & $1.3(0.5-10.4)$ & $1.6(0.3-3.7)$ & $0.7(0.0-3.4)$ \\
\hline Verrucomicrobia & $0.4(0.1-7.3)$ & $0.5(0.1-2.5)$ & $0.4(0.0-7.8)$ \\
\hline Fusobacteria & $0.3(0.0-1.5)$ & $0.3(0.0-5.7)$ & $0.2(0.0-3.5)$ \\
\hline Spirochaetes & $0.1(0.0-3.7)$ & $0.1(0.0-1.2)$ & $0.1(0.0-2.13)$ \\
\hline Fibrobacteres & $0.0(0.0-1.1)$ & $0.0(0.0-0.7)$ & $0.0(0.0-0.8)$ \\
\hline \multicolumn{4}{|l|}{ Class $(10 / 25)^{a}$} \\
\hline Clostridia & $35.8(13.1-59.3)$ & $41.1(20.1-70.9)$ & $38.6(17.8-68.0)$ \\
\hline Negativicutes & $15.4(5.1-36.1)$ & $9.0(0.1-43.3)$ & $14.6(1.3-39.1)$ \\
\hline Eryspelotrichia & $16.8(0.72-33.3)$ & $10.6(0.3-25.3)$ & $7.7(0.4-15.0)$ \\
\hline Bacilli & $8.2(2.4-33.3)$ & $9.8(2.1-32.0)$ & $6.2(0.6-29.8)$ \\
\hline Actinobacteria & $2.9(0.8-32.8)$ & $2.5(0.4-30.1)$ & $4.0(0.5-13.6)$ \\
\hline Gammaproteobacteria & $2.9(0.8-17.0)$ & $4.7(1.0-16.0)$ & $4.4(0.1-35.8)$ \\
\hline Bacteria_unclassified & $1.3(0.5-10.4)$ & $1.6(0.3-3.7)$ & $1.7(0.0-6.4)$ \\
\hline Bacteroidia & $1.5(0.4-4.7)$ & $1.6(0.4-3.6)$ & $1.5(0.3-5.1)$ \\
\hline Firmicutes_unclassified & $0.4(0.1-4.2)$ & $0.4(0.1-1.8)$ & $0.5(0.0-5.3)$ \\
\hline Alphaproteobacteria & $0.9(0.4-4.0)$ & $1.0(0.4-3.4)$ & $0.8(0.0-3.6)$ \\
\hline \multicolumn{4}{|l|}{ Order $(15 / 56)^{\mathrm{a}}$} \\
\hline Clostridiales & $35.6(12.9-59.3)$ & $40.7(20.1-70.9)$ & $38.2(17.7-68.0)$ \\
\hline Selenomonadales & $15.4(5.1-36.1)$ & $9.0(0.1-43.3)$ & $14.6(1.3-39.1)$ \\
\hline Erysipelotrichales & $16.8(0.7-33.3)$ & $10.6(0.3-25.3)$ & $7.7(0.4-15.0)$ \\
\hline Lactobacillales & $4.7(1.2-15.3)$ & $5.8(1.2-14.8)$ & $5.2(0.3-14.3)$ \\
\hline Bacillales & $3.0(0.7-17.9)$ & $3.7(0.9-17.1)$ & $2.2(0.2-17.3)$ \\
\hline Bifidobacteriales & $0.9(0.4-29.0)$ & $0.7(0.1-24.9)$ & $1.3(0.2-11.9)$ \\
\hline Coriobacteriales & $1.1(0.3-4.8)$ & $1.3(0.2-5.1)$ & $1.4(0.2-7.4)$ \\
\hline Bacteria_unclassified & $1.3(0.5-10.4)$ & $1.6(0.3-3.7)$ & $1.7(0.0-6.4)$ \\
\hline Bacteroidales & $1.5(0.38-4.7)$ & $1.6(0.4-3.6)$ & $1.5(0.3-5.1)$ \\
\hline Aeromonadales & $0.2(0.0-9.4)$ & $0.9(0.0-6.9)$ & $0.6(0.0-32.0)$ \\
\hline Enterobacteriales & $0.5(0.1-1.6)$ & $0.7(0.1-10.6)$ & $0.7(0.0-4.5)$ \\
\hline Firmicutes_unclassified & $0.4(0.1-4.2)$ & $0.4(0.1-1.8)$ & $0.5(0.0-5.3)$ \\
\hline Verrucomicrobiales & $0.2(0.1-1.6)$ & $0.3(0.1-0.9)$ & $0.3(0.0-7.5)$ \\
\hline Campylobacterales & $0.4(0.0-10.5)$ & $0.3(0.0-3.1)$ & $0.4(0.0-3.6)$ \\
\hline Xanthomonadales & $0.3(0.1-1.0)$ & $0.4(0.1-1.0)$ & $0.4(0.0-7.1)$ \\
\hline \multicolumn{4}{|l|}{ Family $(20 / 99)^{a}$} \\
\hline Peptostreptococcaceae & $13.1(4.1-32.5)$ & $16.5(0.6-53.0)$ & $12.8(0.3-50.7)$ \\
\hline Veilloncellaceae & $14.2(4.5-36.1)$ & $8.8(0.1-40.8)$ & $14.3(0.4-39.1)$ \\
\hline Erysipelotrichaceae & $16.8(0.7-33.3)$ & $10.6(0.3-25.3)$ & $7.7(0.4-15.0)$ \\
\hline Lachnospiraceae & $9.7(2.7-23.1)$ & $9.7(6.2-23.2)$ & $8.4(3.8-17.0)$ \\
\hline Ruminococcaceae & $3.6(1.2-13.1)$ & $3.4(1.2-9.7)$ & $5.9(0.7-11.6)$ \\
\hline
\end{tabular}


Table 3 Relative abundances of the fecal microbiota members of healthy lean cats and obese cats before and after weight loss (Continued)

\begin{tabular}{|c|c|c|c|}
\hline & \multicolumn{3}{|c|}{ Medians (Min-Max) of relative abundance (\%) } \\
\hline & LEAN & OBWL & OAWL \\
\hline Bifidobacteriaceae & $0.9(0.4-29.0)$ & $0.7(0.1-24.9)$ & $1.3(0.2-11.9)$ \\
\hline Clostridiales_unclassified & $1.2(0.4-3.4)$ & $1.3(0.2-4.4)$ & $1.5(0.5-11.1)$ \\
\hline Clostridiaceae_1 & $0.3(0.1-5.7)$ & $0.6(0.1-16.6)$ & $1.4(0.1-18.6)$ \\
\hline Coriobacteriaceae & $1.1(0.3-4.8)$ & $1.3(0.2-5.1)$ & $1.4(0.2-7.4)$ \\
\hline Enterococcaceae & $1.9(0.2-12.0)$ & $2.3(0.3-11.6)$ & $1.5(0.1-9.4)$ \\
\hline Succinivibrionaceae & $0.2(0.0-9.3)$ & $0.9(0.0-6.8)$ & $0.4(0.0-32.0)$ \\
\hline Planococcaceae & $1.4(0.3-8.0)$ & $2.2(0.6-8.3)$ & $1.2(0.1-8.3)$ \\
\hline Lactobacillaceae & $0.8(0.0-6.3)$ & $0.5(0.1-11.2)$ & $0.8(0.1-4.6)$ \\
\hline Enterobacteriaceae & $0.5(0.1-4.6)$ & $0.7(0.1-10.6)$ & $0.7(0.0-4.5)$ \\
\hline Petptococcaceae_1 & $1.3(0.0-3.5)$ & $0.4(0.0-5.3)$ & $0.7(0.0-4.1)$ \\
\hline Firmicutes_unclassified & $0.4(0.1-4.2)$ & $0.4(0.1-1.8)$ & $0.5(0.0-5.3)$ \\
\hline Bacteroidaceae & $0.9(0.2-2.7)$ & $0.7(0.2-3.1)$ & $1.0(0.1-2.8)$ \\
\hline Acidaminococcaceae & $0.7(0.1-2.3)$ & $0.3(0.0-2.5)$ & $0.2(0.0-3.8)$ \\
\hline Verrucomicrobiaceae & $0.2(0.1-1.6)$ & $0.3(0.0-7.5)$ & $0.3(0.1-0.9)$ \\
\hline \multicolumn{4}{|l|}{ Genera $(20 / 199)^{a}$} \\
\hline Clostridium_XI & $12.3(3.9-32.4)$ & $15.5(0.5-51.3)$ & $12.5(0.3-50.6)$ \\
\hline Megasphaera & $9.6(0.0-35.4)$ & $6.2(0.0-32.6)$ & $9.3(0.0-38.8)$ \\
\hline Erysipelotrichaceae_incertae_sedis & $8.0(0.4-33.0)$ & $8.3(0.1-18.4)$ & $5.2(0.2-12.7)$ \\
\hline Lachnospiraceae & $3.5(1.1-7.0)$ & $3.7(1.9-7.6)$ & $3.0(1.6-8.6)$ \\
\hline Blautia & $3.5(0.9-13.0)$ & $4.1(1.4-7.7)$ & $2.7(0.6-8.5)$ \\
\hline Bifidobacterium & $0.9(0.4-29.0)$ & $0.7(0.1-24.9)$ & $1.3(0.2-11.9)$ \\
\hline Megamonas & $2.6(0.0-11.7)$ & $0.8(0.0-15.4)$ & $3.1(0.0-18.4)$ \\
\hline Clostridiales_unclassified & $1.22(0.4-9.4)$ & $1.3(0.2-4.4)$ & $1.5(0.5-11.1)$ \\
\hline Ruminococcaceae & $1.4(0.4-6.8)$ & $1.2(0.3-3.8)$ & $1.3(0.4-5.5)$ \\
\hline Bacteria_unclassified & $1.3(0.5-10.4)$ & $1.6(0.3-3.7)$ & $1.7(0.0-6.4)$ \\
\hline Clostridium_sensu_stricto & $0.2(0.0-1.4)$ & $0.2(0.1-15.9)$ & $0.6(0.1-18.3)$ \\
\hline Collinsella & $0.8(0.2-3.4)$ & $0.9(0.2-2.2)$ & $0.9(0.1-6.7)$ \\
\hline Enterococcus & $1.6(0.2-10.7)$ & $2.0(0.3-10.4)$ & $1.4(0.1-8.4)$ \\
\hline Erysipelotrichaceae & $3.0(0.0-6.7)$ & $0.2(0.0-4.9)$ & $0.3(0.0-5.0)$ \\
\hline Faecalibacterium & $0.8(0.0-2.4)$ & $0.7(0.1-3.6)$ & $0.8(0.0-7.0)$ \\
\hline Anaerobiospirillum & $0.0(0.0-8.5)$ & $0.0(0.0-6.8)$ & $0.1(0.0-31.9)$ \\
\hline Lactobacillus & $0.7(0.0-6.1)$ & $0.5(0.1-10.6)$ & $0.7(0.1-4.4)$ \\
\hline Planococcaceae & $1.3(0.0-3.5)$ & $0.4(0.0-5.3)$ & $0.7(0.0-4.1)$ \\
\hline Firmicutes_unclassified & $0.4(0.1-4.2)$ & $0.4(0.1-1)$ & $0.5(0.0-5.3)$ \\
\hline Lachnospiraceae_incertae_sedis & $0.9(0.3-2.6)$ & $1.1(0.2-2.7)$ & $1.0(0.1-2.5)$ \\
\hline
\end{tabular}

LEAN lean cats, OBWL obese cats before weight loss, OAWL obese cats after weight loss

The relative abundances across taxa presented here represent the fecal microbiota of healthy lean cats (LEAN, $n=14)$ and obese cats (OBWL, $n=17)$ following a 4-week adaptation period with a veterinary therapeutic food intended for weight loss and adult maintenance, and obese cats after a 10 -week weight loss period on the same food (OAWL, $n=16$ ). Cut-off for phyla and genera in the study were 1 and $0.1 \%$, respectively. However, only a portion of the most abundant members for all taxa (besides to phyla) are presented in the table. No significant differences were found between groups, using Wilcoxon Rank Sum and Wilcoxon Signed-Rank, depending on the groups' comparison, followed by the Benjamini-Hochberg adjustment $(P>0.05$ for all comparisons; not shown in the table) ${ }^{a}$ The numbers after each taxa represent the number of members in the specific taxa presented in the table out of the overall number of members in that taxa retrieved by the analyses 

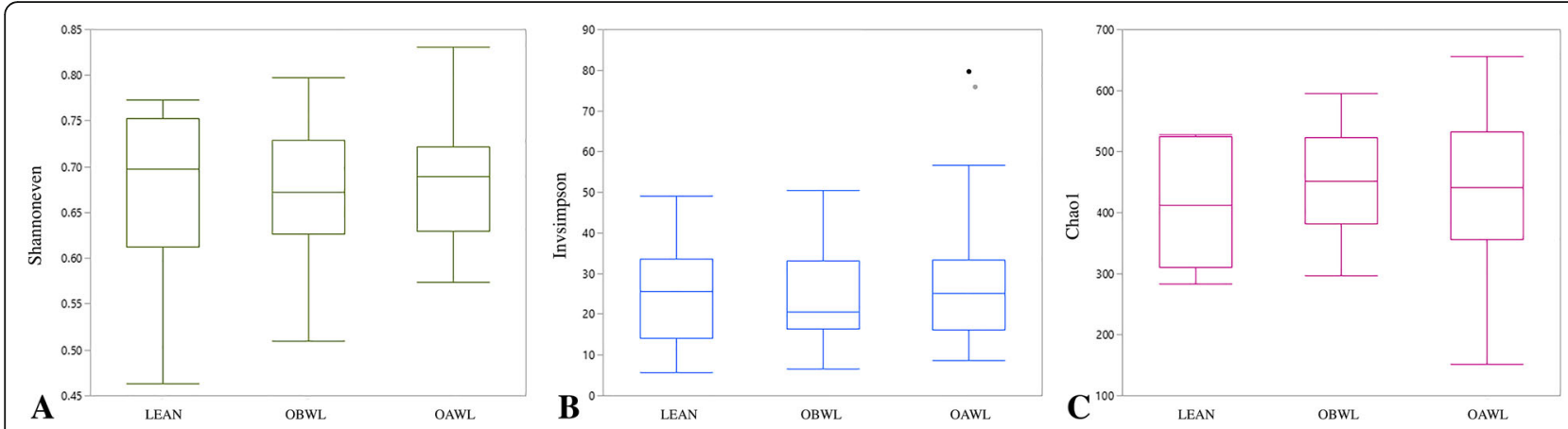

Fig. 3 Bacterial population evenness (Shannoneven), diversity (Invsimpson), and richness (Chao1) in healthy lean cats (LEAN, $n=14$ ) and obese cats (OBWL, $n=17$ ) following a 4-week adaptation period with a veterinary therapeutic food intended for weight loss and adult maintenance, and obese cats after a 10-week weight loss period on the same food (OAWL, $n=16$ )

group to the obese group before energy restriction was observed, and unlike the common literature and the current study, they observed increased Firmicutes abundance in the lean neutered cats [25]. The authors of the mentioned study interpreted these finding as supportive to the idea that alterations in abundance at lower taxonomic level, such as genus, may have more impact and health effects compared with alterations in abundance at higher taxonomic level (e.g. phylum) [25]. The authors of the current study concur with this notion, despite the difference in findings.

In the current study, Planococcaceae incertae sedis, a taxa that belongs to the Planococcaceae family, was found to be enriched in obese cats before weight loss compared to lean cats. Two unclassified members from this family were also found to be enriched in pigs that

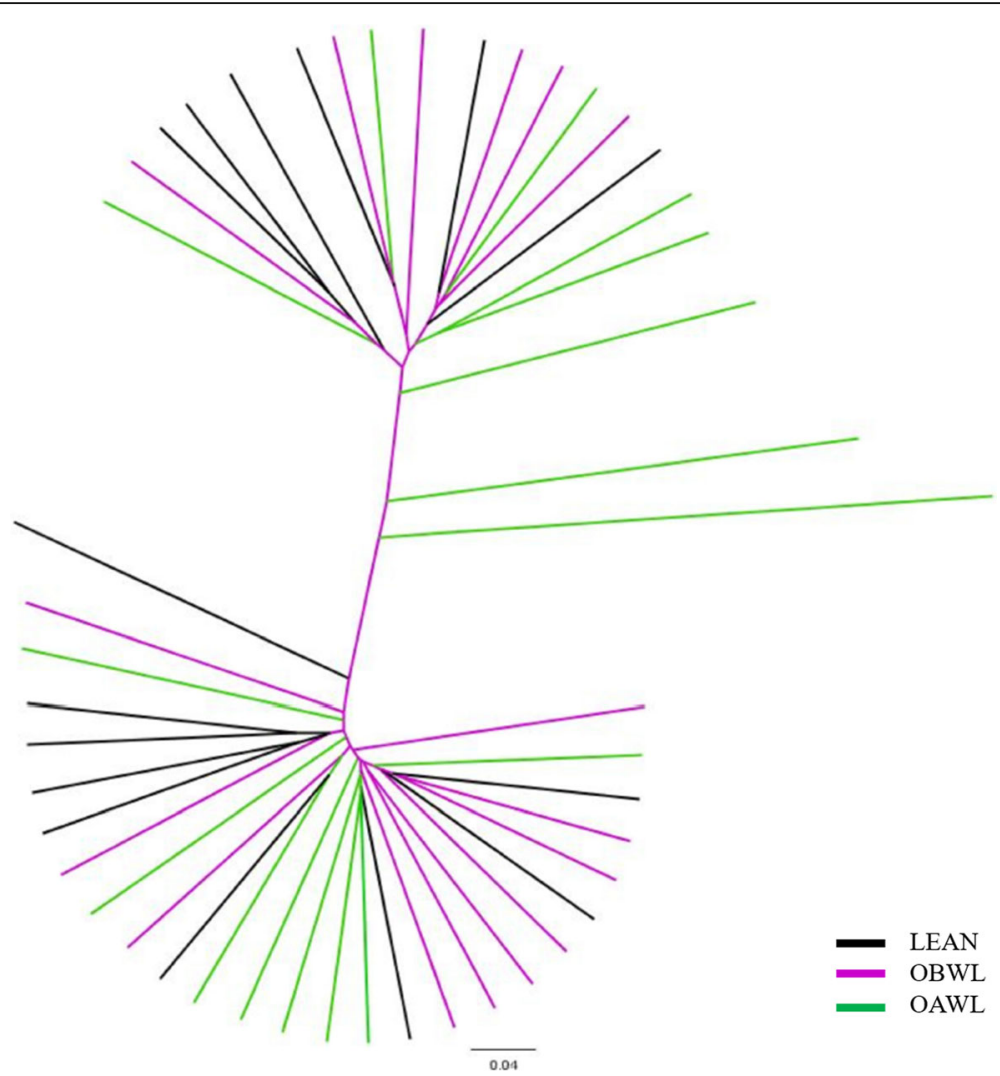

Fig. 4 Dendogram of the Classical Jaccard index representing the community membership of the fecal microbiota in healthy lean cats (LEAN, $n=14)$ and obese cats (OBWL, $n=17)$ following a 4-week adaptation period with a veterinary therapeutic food intended for weight loss and adult maintenance, and obese cats after a 10-week weight loss period on the same food (OAWL, $n=16)$. Each group is represented with a different colour (see legend) 


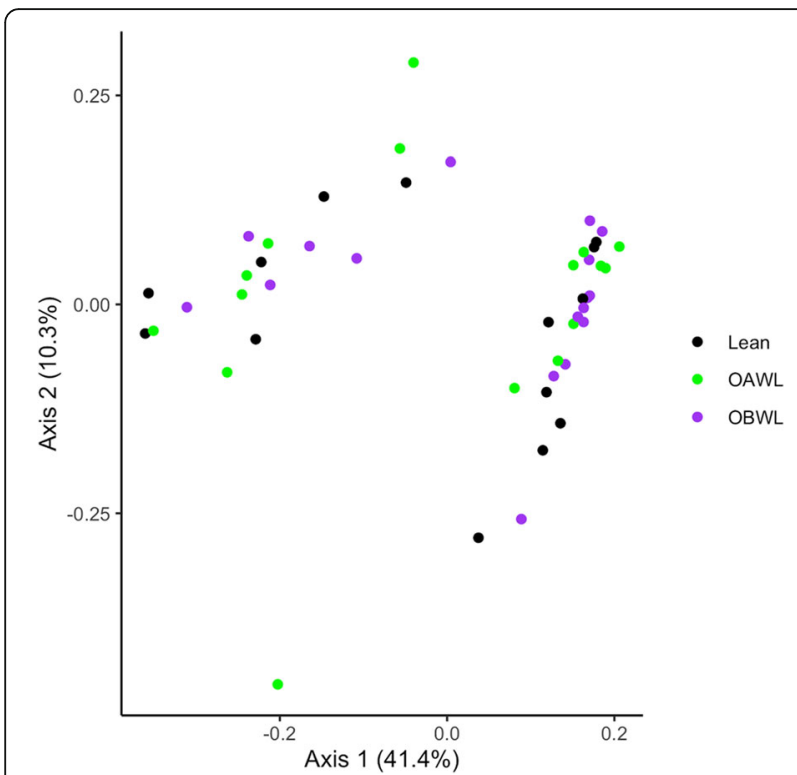

Fig. 5 Three dimensional principal coordinate analysis of population membership of the fecal microbiota of healthy lean cats (LEAN, $n=$ 14) and obese cats (OBWL, $n=17)$ following a 4-week adaptation period with a veterinary therapeutic food intended for weight loss and adult maintenance, and obese cats after a 10-week weight loss period on the same food (OAWL, $n=16)$. Each group is represented with a different colour (see legend)

had a higher residual feed intake, meaning, they were more efficient in energy harvesting [38]. In obese twins, a lower proportion of Bacteroidetes versus Actinobacteria was found, although the abundance of Firmicutes was not different. The same study identified obesityrelated genes from the microbiome and found that $75 \%$ of the enriched obesity-related genes in obese twins originated from Actinobacteria, whereas the residual 25\% originated from Firmicutes. Many of these genes' functions are related to carbohydrate, amino-acid or lipid metabolism [37]. In the current study, Firmicutes, as well as Actinobacteria, were enriched in obese cats before weight loss compared to lean cats. In humans, obesity-specific Prevotellaceae, which belongs to the phylum Bacteroidetes, as well as some families that belong to the phylum Proteobacteria were enriched in morbidly obese individuals compared to individuals who went through gastric bypass or had a normal weight. Enrichment of Prevotellaceae and Proteobacteria was also demonstrated in obese cats before weight loss compared to lean cats enrolled in the current study. The Prevotellaceae family has members that facilitate protein and carbohydrate fermentation, as well as acetate, $\mathrm{H}_{2}$ and folate producers - hence, may have implications on energy metabolism and intestinal health $[39,40]$. At last, in contrast to the current study, Verrucomicrobia was more abundant in individuals with a normal body weight or in individuals who had gastric bypass surgery, rather than in obese people [40]. Nonetheless, further understanding of the interaction between obesity and microbial features in the different species requires metagenomics research to further explore the obesityrelated metabolic pathways and functional potential of the microbiome.

In addition, interesting complementary findings were observed when examining the effect of feline obesity and weight loss on serum cobalamin and folate concentrations. Serum cobalamin concentrations were lower in obese cats both before and after weight loss when compared to lean cats. Also, serum folate concentrations were higher in obese cats before weight loss compared to after weight loss. Both, serum cobalamin and folate concentrations, were within the laboratory's reference interval in all cats, although for folate, concentrations were on the high end of the reference interval for obese cats after weight loss. A correlation between BW and serum cobalamin concentrations could not be demonstrated in a previous feline study [41], while in humans obesity is associated with a risk for cobalamin deficiency [42]. One of the suggested reasons for obesity-associated cobalamin deficiency is the diet consumed by obese humans, which is commonly richer in carbohydrates and fat, and lower in animal-derived protein, i.e., a diet lower in cobalamin [43]. Still, low cobalamin intake was not expected in the current study. First, as strict carnivores, cats consume a diet high in animal-derived protein and therefore also in cobalamin. Second, the cats were fed a complete and balanced diet formulated for adult maintenance and intended for weight loss, meaning that protein and micronutrient content were enhanced to compensate for reduced energy intake. Hence, it is possible that no true cobalamin deficiency was observed due to the consumption of a cobalamin-enriched diet.

Serum cobalamin and folate concentrations are indicators for gastrointestinal disease in humans [44]. In cats and dogs, a similar association has been suggested due to the positive therapeutic outcome of intestinal disease when cobalamin concentrations are replenished [28, 29]. In general, both cobalamin and folate are affected by intestinal health and more specifically intestinal absorptive capacity of various segments of the small intestine (i.e., folate in the proximal small intestine and cobalamin in the ileum) $[26,28,29]$. Considering an association between serum cobalamin and folate and intestinal health and absorption, the current findings, whilst still being within the normal reference interval, may suggest better intestinal properties in lean compared to obese cats and improvement of intestinal health with weight loss.

The presence of enriched taxa in obese cats prior to weight loss, compared to lean cats, as well as the failure to identify such differences in enriched taxa between obese cats before and after weight loss, may 
Table 4 Significantly enriched operational taxonomic units (OTUs) of the fecal microbiota of lean cats and obese cats before and after weight loss

\begin{tabular}{lll}
\hline \multirow{2}{*}{ Comparison } & Significantly enriched OTUs & \\
\cline { 2 - 3 } & LEAN & OBWL \\
\hline LEAN vs. OBWL & Tenericutes & Firmicutes \\
Anaeroplasma & Unclassified Clostridiales \\
& (5 OTUs) & Planococcaceae_incertae_sedis \\
& TM7 & TM7_genus_incertae_Sedis \\
& Actinobacteria & Arthrobacter \\
& Nocardioides \\
& Bacteroidetes \\
& Unclassified \\
& Prevotellaceae \\
& Verrucomicrobia \\
& Unclassified \\
& Verrucomicrobiaceae \\
& Proteobacteria \\
Oligella & Unclassified \\
& Proteobacteria
\end{tabular}

OTUs operational taxonomic units, LEAN lean cats, OBWL obese cats before weight loss, OAWL obese cats after weight loss

The OTUs represented here are from the fecal microbiota of healthy lean cats (LEAN, $n=14$ ) and obese cats (OBWL, $n=17)$ following a 4 -week adaptation period with a veterinary therapeutic food intended for weight loss and adult maintenance, and obese cats after a 10-week weight loss period on the same food (OAWL, $n=16$ ). The presented OTUs have a linear discriminant analysis score $>2$, and are organized in a descending manner

strengthen the findings that body composition and \% weight loss, while excluding diet as a confounder, are the main drivers of changes in the fecal microbiota [34-37]. Although BW and BCS significantly decreased with energy restriction, the weight loss observed may not have been sufficient to cause significant changes in the biodiversity indices, relative abundance or LEfSe analysis in obese cat over the course of the weight loss plan. Still, fewer differences in enriched taxa were observed when comparing obese cats after weight loss to the lean cats. This might mean that with weight loss, the microbial population membership and composition are changing slowly, yet a 10-week weight loss period might not be long enough to completely revert the obesityrelated differences in enriched taxa to a lean body condition.

Challenges occurred with fecal collection during the current study. The owners were advised to maintain the fecal samples at $4{ }^{\circ} \mathrm{C}$ until transfer to the clinic, yet, some owners kept them at ambient temperature. In a study by Weese and Jalali (2014), no changes in microbial membership and composition were observed during 7 day storage at $4{ }^{\circ} \mathrm{C}$ [45]. Likewise, a recent study investigated the effects of storage of fecal samples at ambient temperature on the feline fecal microbiota and confirmed the validity of using feline fecal samples that were kept at an ambient temperature for up to 4days for microbiota-related analyses [46].
Therefore, despite the mentioned challenges, the authors did not expect fecal samples storage conditions to affect the results of the microbial-related analyses.

In the current study, aside to the relatively short weight loss period that was not sufficient for obese cats to reach their ideal body weight, the sample size was relatively small for all groups, and can be considered a limitation. However, since a similar study had not previously been conducted in client-owned cats, the samples size was aligned with other feline studies, investigating the effects of GI inflammatory conditions on the microbial population $[47,48]$. Nevertheless, it is possible that with a larger sample size, and a longer period of caloric restriction would promote greater weight loss as well as more distinct and consistent changes in the fecal microbiota than were identified in the current study here.

\section{Conclusions}

Enriched taxa in LEfSe analysis were observed especially when comparing obese cats before and after weight loss. Nearly half of the enriched taxa in the obese group belonged to Firmicutes, which concurs with previous reports in humans and mice. This may be related to better energy-harvesting abilities of the host. However, a metagenomic approach is warranted to explore the functional potential of the feline obese fecal microbiome. The current study also demonstrated minimal effects of a 10week standardized and successful weight loss plan on 
microbial biodiversity, while excluding diet as a confounder. This may imply that sudden short-term energy restriction is not enough to revert microbial changes in the intestines.

Moreover, assessment of serum cobalamin and folate, although all concentrations were within the reference interval, potentially suggest improved intestinal health in lean compared to obese cats, which could be achieved with weight loss in obese cats. Nevertheless, more research is warranted allowing for a longer weight loss period and a larger sample size.

\section{Methods}

\section{Experimental design}

Fourteen lean (BCS 4-5/9, 10 males and 4 females) and 17 obese (BCS $\geq 8 / 9,11$ males and 6 females) cats were enrolled into the study [49]. All cats were client-owned animals from the Guelph, Ontario region, who live indoors, were neutered and between 2 and 9 years of age. All cats were assessed to be healthy, apart from obesity, based on physical examination and medical history provided by the cat's owner, as well as complete blood count $(\mathrm{CBC})$ and serum biochemistry profile. There was no history of antimicrobial or antiinflammatory medications administered in the 90 day period before commencing study enrolment. The study took place between May 2015 and December 2016.

\section{Adaptation period}

At the time of enrolment, BW, BCS and muscle condition score (MCS) $[49,50]$ were documented. Next, all cats underwent a one-week transition period to a veterinary therapeutic food (Hill's Prescription Diets Metabolic Feline dry, Hill's Pet Nutrition, Topeka, Kansas, USA) intended for weight loss and adult maintenance (Table 5), followed by a 4-week adaptation period during which all cats received the study food for $100 \%$ of their daily ration. Individual maintenance energy requirements (ER) for both lean cats (LEAN) and OBWL were calculated in accordance with the National Research Council (NRC) (LEAN: $100 \mathrm{Kcal} / \mathrm{kg}^{0.67}$; OBWL: 130 $\mathrm{Kcal} / \mathrm{kg}^{0.4}$ ) based on ideal body weights [51]. The owners recorded the daily food intake of each cat. Three weeks following study enrolment, body weight was assessed, and the amount of food offered was adjusted, aiming to maintain a stable body weight. At the end of the adaptation period (week 5) BW, BCS and MCS were recorded. Body condition score was once again evaluated using a 9-point scale, previously validated for cats [49]. Muscle condition score was assessed using a 4-point scale, which was previously described and validated for cats [52]. Also, BMI and girth were assessed in all cats at that time. Body mass index was calculated according to a previous study [53] and girth was measured right behind
Table 5 Proximate and total dietary fibre analyses of the veterinary therapeutic food fed in this study ${ }^{a}$

\begin{tabular}{lll}
\hline & Units & Content $^{\mathrm{b}}$ \\
\hline Moisture & $\mathrm{g} / 100 \mathrm{~g}$ & 5.5 \\
CF (by acid hydrolysis) (DM) & $\mathrm{g} / 100 \mathrm{~g}$ & 13.0 \\
CP (DM) & $\mathrm{g} / 100 \mathrm{~g}$ & 38.6 \\
$\mathrm{NFE}(\mathrm{DM})^{\mathrm{c}}$ & $\mathrm{g} / 100 \mathrm{~g}$ & 36.3 \\
Cf (Cf) (DM) & $\mathrm{g} / 100 \mathrm{~g}$ & 6.3 \\
Total dietary fibre (DM) & $\mathrm{g} / 100 \mathrm{~g}$ & 18.5 \\
CA (DM) & $\mathrm{g} / 100 \mathrm{~g}$ & 5.8 \\
Energy density (DM) $^{\mathrm{d}}$ & $\mathrm{kcal} / 100 \mathrm{~g}$ & 372.7 \\
\hline
\end{tabular}

$C F$ crude fat, $C P$ crude protein, NFE nitrogen-free extract, $C f$ crude fibre, $D M$ dry matter, $C A$ crude ash

The food was fed as the only food source to lean cats (LEAN, $n=14$ ) for adult maintenance for 4 weeks and to obese cats for adult maintenance for 4 weeks (OBWL, $n=17$ ), followed by a 10-week weight loss period (OAWL, $n=16$ ) aHill's Prescription Diets Metabolic Feline (dry), which contained chicken byproduct meal, brewers rice, corn, gluten meal, powdered cellulose, dried tomato, pomace, flaxseed, dried beet pulp, chicken liver flavor, coconut oil, pork fat, lactic acid, potassium chloride, calcium sulfate, L-lysine, choline chloride, carrots, DL-methionine, vitamins (vitamin E supplement, L-ascorbyl-2polyphosphate (source of vitamin C), niacin supplement, thiamine mononitrate, calcium pantothenate, pyridoxine hydrochloride, vitamin $\mathrm{A}$ supplement, riboflavin supplement, biotin, vitamin B12 supplement, folic acid, vitamin D3 supplement), taurine, L-carnitine, minerals (manganese sulfate, ferrous sulfate, zinc oxide, copper sulfate, calcium iodate, sodium selenite), mixed tocopherols for freshness, natural flavors, $\beta$-carotene

${ }^{b}$ Nutrient content refers to an average of two consecutive laboratory analyses from the same bag, which were performed by Maxxam Analytics International Corporation, Mississauga, Ontario, Canada;

${ }^{C}$ Calculated using the equation: NFE $(g / 100 \mathrm{~g})=100-(\mathrm{CP}+\mathrm{CF}+\mathrm{Cf}+\mathrm{CA})[51]$

${ }^{\mathrm{d} C a l c u l a t e d}$ using the equation: Energy density

$(\mathrm{kcal} / 100 \mathrm{~g})=(\mathrm{CF} \times 8.5)+(\mathrm{CP} \times 3.5)+(\mathrm{NFE} \times 3.5)[51]$

the last rib [54]. The same investigator (MT) conducted all measurements throughout the study to reduce variability. A blood sample was drawn from the jugular or cephalic vein for analysis of serum cobalamin and folate concentration. Fecal samples were collected by the owners and were obtained by the study personnel within $24 \mathrm{~h}$ after defecation and frozen at $-80^{\circ} \mathrm{C}$ until further analysis. The lean cats completed the study at week 5 , while the obese cats continued with a 10 -week weight loss plan.

\section{Weight loss period}

At week 5, the individual ER for weight loss were calculated for the obese cats in accordance with NRC recommendations $\left(0.6 \times 130 \mathrm{Kcal} / \mathrm{kg}^{0.4}\right)$, based on ideal body weight. The weight loss period continued for 10 weeks. During this period, food intake was recorded daily by the owners and BW, BCS and MCS were assessed every other week to monitor for effective and safe weight loss (with a target BW loss of 0.5 to $2 \%$ of the initial BW per week $[9,55])$, as well as to monitor maintenance of lean body mass. If the weekly weight loss rate was below $0.5 \%$ or exceeded $2 \%$ of the initial BW, individual ER adjustments were made - lowering or increasing the ER initially by $5 \%$, respectively [12]. After 10 weeks, assessment 
of BMI and girth were repeated. At that time, additional blood samples were collected, food logs obtained from the owners. Fecal samples were also collected by the owners for the obese cats after weight loss (OAWL) and fecal samples were stored as described above.

\section{Laboratory analyses of blood samples}

Blood was collected at the end of the adaptation period (LEAN and OBWL) and at the end of the weight loss period (OAWL) for analyses of serum concentrations of cobalamin and folate was refrigerated at $4{ }^{\circ} \mathrm{C}$ for $2 \mathrm{~h}$ and then centrifuged at $3000 \mathrm{rpm}$ for $10 \mathrm{~min}$ (Sorvall Legend RT Centrifuge. Fisher Scientific, Ottawa, Canda). The serum retrieved was aliquoted and frozen at $-80{ }^{\circ} \mathrm{C}$. Frozen serum samples were batched from both the lean and obese groups, and were analyzed together. Serum concentrations of cobalamin and folate were analyzed at the Gastrointestinal Laboratory, Texas A\&M University, using a chemiluminescent enzyme immunoassay (Immulite 2000 Immunoassay System. Siemens Healthcare Systems $\mathrm{GmbH}$, Erlangen, Germany) involving an automated alkaline denaturation procedure.

\section{Sample preparation and DNA extraction}

All fecal samples, collected at the end of the adaptation period (LEAN and OBWL) and at the end of the weight loss period (OAWL), were analysed simultaneously. Whole fecal samples were thawed overnight in their original container in a refrigerator $\left(+4{ }^{\circ} \mathrm{C}\right)$, manually homogenized in a biosafety cabinet (Class II, Type A2 Biosafety Cabinet, Thermo Fisher Scientific, Waltham, Massachusetts, USA) and aliquoted into $200 \mathrm{mg}$ samples.

DNA extraction was conducted using a commercial stool extraction kit (E.Z.N.A. Stool DNA Kit, Omega Bio-Tek Inc., Doraville, Georgia, USA) in accordance with the manufacturer's instructions. Extracted DNA samples were stored at $-80{ }^{\circ} \mathrm{C}$ until further analysis.

\section{Polymerase chain reaction (PCR)}

A spectrophotometer (NanaDrop 1000 Spectrophotometer, Nano Drop Technologies Inc. (Thermo Fisher Scientific), Waltham, Massachusetts, USA) was used to assess the quantity of extracted DNA. All DNA samples were diluted (if needed) to a range of 30 to $100 \mathrm{ng} / \mathrm{ml}$. The V4 region of the $16 \mathrm{~S}$ rRNA gene was amplified using a PCR with the forward primer: S-D-Bact-0564-a-S-15 (5'AYTGGGYDTAAAGNG-3'), reverse: S-D-Bact-0785-bA-18 (5' -TACNVGGGTATCTAATCC-3') [56], KAPA HiFi ReadyMix (Kapa Biosystems, Wilmington, Massachusetts, USA), and PCR grade water. The PCR products were purified with Agencourt AMPure XP (Beckman Coulter Inc., Mississauga, ON, Canada). In order to prepare the PCR products for Illumina MiSEq (Illumina, San Diego, California, USA) sequencing, the purified PCR products were amplified by PCR with Illumina adapters (Mastercycler Pro, Eppendorf Canada Ltd., Mississauga, Ontario, Canada) and then once again purified. Prior to Illumina sequencing, the finalized PCR products were evaluated using gel electrophoresis and DNA was measured using spectrophotometry.

\section{DNA sequencing}

Bridge amplification was performed with an Illumina MiSeq system (Illumina, San Diego, California, USA), using terminator nucleotides that were incorporated into the amplified PCR products with the removal of the terminator group [57].

\section{Bioinformatics and statistical analyses}

Following DNA sequencing of fecal samples, Mothur v1.36.1 was used for sequence processing [58, 59]. Assembly of paired end reads was performed using the make.contigs command. This command extracts the sequence and creates its reverse compliment and joins the reads into contigs. Next, filtration was conducted using several screen.seqs commands to remove sequences greater than $250 \mathrm{bp}$ in length and those with any ambiguous base calls or runs of homopolymers greater than $8 \mathrm{bp}$. Alignment of sequences to the Silva v128 16S rRNA reference database [60] was implemented, with the removal of sequences that did not align with the correct region. Uchime was conducted using the same Silva reference database to identify chimeras [61], which were then removed. Archaea were removed as well using the remove. Lineage command. A closed OTU picking approach was then used. Ribosomal Database Project (RDP) classifier (v14) was used for taxonomic assignment of sequences [62], and the reference database used was v11.4. In order to standardize sequence numbers used for analysis, subsampling was completed based on the smallest number of sequences from a sample [63].

Further statistical analyses were performed using JMP 13.0 (SAS Campus Drive, Cary, North Carolina, USA). Normality of data distribution was assessed using the Shapiro-Wilk test. Evenness, diversity and richness were calculated using Shannon diversity [64], Simpson diversity [65] and Chao [66] respectively, and a nonparametric multiple comparison test (Wilcoxon Rank Sum) was used to compare between the groups and time points (LEAN, OBWL and OAWL). Relative abundances were calculated for the different taxonomic levels, for each group. The relative abundance threshold for phyla was above $1 \%$ and above $0.1 \%$ for the rest of the taxa levels. Differences were evaluated using nonparametric multiple comparison test (Wilcoxon Rank Sum for comparisons between groups: LEAN to OBWL and LEAN to OAWL; Wilcoxon SignedRank for the comparison between time points: OBWL to OAWL), with $p$-values adjusted using the Benjamini- 
Hochberg correction (SAS Campus Drive, Cary, North Carolina, USA; R. Core Team, 2013, R Foundation for Statistical Computing, Vienna, Austria) to control for false discovery [67]. Relative abundances are presented as median with range (minimum to maximum). The classical Jaccard index [68] and Yue \& Clayton index of dissimilarity [69] (beta-diversity indexes) were calculated to examine community membership and population structure, respectively. To reflect the differences in membership and structure between the groups, dendrograms were generated, and significance of clustering according to group was determined using parsimony and unweighted unifrac tests [70]. Beta-diversity indices were also visualized using principal coordinate analyses ( $\mathrm{PCoA})$, with further comparison of groups by AMOVA and HOMOVA. In order to identify difference in taxa between all groups, linear discriminatory analysis (LDA) effective size (LefSe) [71] was conducted.

Statistical analyses for body weight, body composition measurements and serum cobalamin and folate concentrations were performed using SAS v9.3 (SAS Campus Drive, Cary, North Carolina, USA). Normality of the data was assessed using the Shapiro-Wilk test. Data that did not distribute normally were logged, and if the distribution was still not normal, then non-parametric tests were performed. Differences in BW, BCS, BMI and girth measurements, as well as serum cobalamin and folate concentrations between LEAN to OBWL and OAWL groups were compared using a student T-test/paired Ttest or the corresponding Wilcoxon-Mann-Whitney/ Wilcoxon Signed-Rank, depending on normality and on whether the samples were paired. Significance was set at $P<0.05$ for all comparisons. Normally distributed data are expressed as mean SD (BW, BMI, girth measurements) or as mean of the back transformed values (lower limit (LL)-upper limit (UL)) (folate). Data that did not have a normal distribution are presented as median with range (minimum to maximum) (cobalamin, $\mathrm{BCS}$ ).

\section{Abbreviations}

Gl: Gastrointestinal; PCR: Polymerase chain reaction; LPS: Lipopolysaccharide; OBWL: Obese cats before weight loss; OAWL: Obese cats after weight loss; BW: Body weight; SD: Standard deviation; BCS: Body condition score; BMI: Body mass index; OUT: Operational taxonomic unit; LEfSe: Linear discriminatory analysis effective size; SCFA: Short chain fatty acids; CBC: Complete blood count; MCS: Muscle condition score; ER: Energy requirements; RDP: Ribosomal Database Project; PCoA: Principal coordinate analyses; HOMOVA: Homogeneity of molecular variance; AMOVA: Analysis of molecular variance; LDA: Linear discriminatory analysis; LL: Lower limit; UL: Upper limit
}

\section{Acknowledgements}

We wish to thank cat owners and their cats for participating in the study, as well as many undergraduate volunteers, graduate students, and technicians that helped with sample collection. We also wish to thank Charlotte Chau and Bianca Di Sabatino for assisting with sample analyses, Joyce Rousseau for her technical assistance with the laboratory work, Maisie McDowell for assistance with electronic artwork and Allison Lull for manuscript formatting.
This research was previously presented at the 18th Annual AAVN Clinical Nutrition and Research Symposium June 13, 2018, Seattle, WA, USA. (https:// doi.org/10.1111/jpn.13043).

\section{Authors' contributions}

Designed the experiment: AV, JSW, MH, and MT. Performed the experiment: MT. Supervised serum folate and cobalamin analyses: JMS. Analysed the data: MT, DEG, and JSW. Drafted the manuscript: MT. All authors contributed to manuscript preparation and approved the final manuscript.

\section{Authors' information}

The study is within the scope of the graduate studies, Doctor Veterinary Science (DVSC) degree, of MT, who received a DVSc scholarship from the Ontario Veterinary College, University of Guelph.

\section{Funding}

The research was supported by Hill's Pet Nutrition Inc. and by a Natural Sciences Engineering Research Council of Canada, Discovery Grant. The funders had no role in the design of the study or in the collection, analysis and interpretation data or in writing the manuscript. Hill's Pet Nutrition Inc. reviewed and approved the manuscript before submission for publication.

\section{Availability of data and materials}

The dataset generated and/or analysed during the current project is available at the Scholars Portal Dataverse server (https://dataverse. scholarsportal.info/dataset.xhtml?persistentld=doi:10.5683/SP/9VAK4K).

\section{Ethics approval and consent to participate}

The study was conducted at the Ontario Veterinary College, University of Guelph. The experimental design was approved by the University of Guelph Animal Care Committee (Animal Utilization Protocol \#2496), and prior to study enrolment, cat owners signed informed consent forms for their cat's participation.

\section{Consent for publication}

Not applicable.

\section{Competing interests}

All authors declare no conflict of interest. A.V. is the Royal Canin Veterinary Diets Endowed Chair in Canine and Feline Clinical Nutrition at the Ontario Veterinary College.

\section{Author details}

${ }^{1}$ Department of Clinical Studies, Ontario Veterinary College, University of Guelph, Guelph, ON N1G 2W1, Canada. ${ }^{2}$ Present address: Royal Canin Canada, 100 Beiber Rd, NOB 2J0 Puslinch, Canada. ${ }^{3}$ Department of Pathobiology, Ontario Veterinary College, University of Guelph, Guelph, ON N1G 2W1, Canada. ${ }^{4}$ Laboratory of Animal Nutrition, Faculty of Veterinary Medicine, Ghent University, Merelbeke B-9820, Belgium. ${ }^{5}$ Gastrointestinal Laboratory, Department of Small Animal Clinical Sciences, College of Veterinary Medicine and Biomedical Sciences, Texas A\&M University, College Station 77843, TX, USA.

Received: 15 May 2019 Accepted: 16 March 2020

Published online: 15 April 2020

\section{References}

1. German A. Obesity in companion animals. In Practice. 2010:32(2):42-50.

2. Laflamme DP. Companion animals symposium: obesity in dogs and cats: what is wrong with being fat? J Anim Sci. 2012;90(5):1653-62.

3. Laflamme DP. Understanding and managing obesity in dogs and cats. Vet Clin North Am Small Anim Pract. 2006:36(6):1283-95.

4. Chandler M, Cunningham S, Lund EM, Khanna C, Naramore R, Patel A, Day MJ. Obesity and associated comorbidities in people and companion animals: a one health perspective. J Comp Pathol. 2017;156(4):296-309.

5. Courcier EA, Mellor DJ, Pendlebury E, Evans C, Yam PS. An investigation into the epidemiology of feline obesity in Great Britain: results of a crosssectional study of 47 companion animal practises. Vet Rec. 2012;171(22):560.

6. U.S. Pet Obesity Rates Plateau and Nutritional Confusion Grows [https:// petobesityprevention.org/2018]. Accessed December 2019. 
7. Cave NJ, Allan FJ, Schokkenbroek SL, Metekohy CA, Pfeiffer DU. A cross-sectional study to compare changes in the prevalence and risk factors for feline obesity between 1993 and 2007 in New Zealand. Prev Vet Med. 2012;107(1-2):121-33.

8. Colliard L, Paragon BM, Lemuet B, Bénet JJ, Blanchard G. Prevalence and risk factors of obesity in an urban population of healthy cats. J Feline Med Surg. 2009;11(2):135-40.

9. Burkholder WJ, Toll PW. Obesity. In: Hand MS, Thatcher CD, Remillard RL, Roudebush PBJ, editors. Small Animal Clinical Nutrition. 4th ed. Marceline: Mark Morris Institute; 2000. p. 401-30.

10. Robertson ID. The influence of diet and other factors on owner-perceived obesity in privately owned cats from metropolitan Perth, Western Australia. Prev Vet Med. 1999;40(2):75-85.

11. McGreevy PD, Thomson PC, Pride C, Fawcett A, Grassi T, Jones B. Prevalence of obesity in dogs examined by Australian veterinary practices and the risk factors involved. Vet Rec. 2005;156(22):695-702.

12. Toll PW, Yamka RM, Schoenherr WD, Hand MS. Obesity. In: Hand MS, Thatcher CD, Remillard RL, Roudebush P, Novotny BJ, editors. Small Animal Clinical Nutrition. 5th ed. Topeka: Mark Morris Institute; 2010. p. 501-41.

13. Bäckhed F, Ding H, Wang T, Hooper LV, Koh GY, Nagy A, Semenkovich CF, Gordon Jl. The gut microbiota as an environmental factor that regulates fat storage. Proc Natl Acad Sci U S A. 2004;101(44):15718-23.

14. Cummings JH, Pomare EW, Branch WJ, Naylor CP, Macfarlane GT. Short chain fatty acids in human large intestine, portal, hepatic and venous blood. Gut. 1987;28(10):1221-7.

15. Schwiertz A, Taras D, Schafer K, Beijer S, Bos NA, Donus C, Hardt PD. Microbiota and SCFA in lean and overweight healthy subjects. Obesity. 2010;18(1):190-5.

16. Hooper LV, Wong MH, Thelin A, Hansson L, Falk PG, Gordon JI. Molecular analysis of commensal host-microbial relationships in the intestine. Science. 2001;291(5505):881-4.

17. Cani PD, Amar J, Iglesias MA, Poggi M, Knauf C, BasFtelica D, Neyrinck AM, Fava F, Tuohy KM, Chabo C. Metabolic endotoxemia initiates obesity and insulin resistance. Diabetes. 2007;56(7):1761-72.

18. Ley RE, Backhed F, Turnbaugh P, Lozupone CA, Knight RD, Gordon Jl. Obesity alters gut microbial ecology. Proc Natl Acad Sci U S A. 2005;102(31):11070-5.

19. Ley RE, Turnbaugh PJ, Klein S, Gordon Jl. Microbial ecology: human gut microbes associated with obesity. Nature. 2006;444(7122):1022-3.

20. Cotillard A, Kennedy SP, Kong LC, Prifti E, Pons N, Le Chatelier E, Almeida M, Quinquis B, Levenez F, Galleron N, et al. Dietary intervention impact on gut microbial gene richness. Nature. 2013;500(7464):585-8.

21. Turnbaugh PJ, Gordon JI. The core gut microbiome, energy balance and obesity. J Physiol. 2009;587(17):4153-8.

22. Handl S, German AJ, Holden SL, Dowd SE, Steiner JM, Heilmann RM, Grant RW, Swanson KS, Suchodolski JS. Faecal microbiota in lean and obese dogs. FEMS Microbiol Ecol. 2013;84(2):332-43.

23. Kieler IN, Mølbak L, Hansen LL, Hermann-Bank ML, Bjornvad CR. Overweight and the feline gut microbiome - a pilot study. J Anim Physiol Anim Nutr. 2016;100(3):478-84.

24. Pallotto MR, de Godoy MRC, Holscher HD, Buff PR, Swanson KS. Effects of weight loss with a moderate-protein, high-fiber diet on body composition, voluntary physical activity, and fecal microbiota of obese cats. Am J Vet Res. 2018;79(2):181-90.

25. Fischer MM, Kessler AM, Kieffer DA, Knotts TA, Kim K, Wei A, Ramsey JJ, Fascetti J. Effects of obesity, energy restriction and neutering on the faecal microbiota of cats. Br J Nutr. 2017:118:513-24.

26. Teixeira TFS, Collado MC, Ferreira CLLF, Bressan J, Peluzio MDCG. Potential mechanisms for the emerging link between obesity and increased intestinal permeability. Nut Res. 2012;32(9):637-47.

27. Brun P, Castagliuolo I, Di Leo V, Buda A, Pinzani M, Palù G, Martines D. Increased intestinal permeability in obese mice: new evidence in the pathogenesis of nonalcoholic steatohepatitis. Am J Physiol Gastrointest Liver Physiol. 2007;292(2):G518-25.

28. Batt R. Update on the non-invasive monotoring of intestinal disease in dogs and cats [folate and cobalamin assay, intestinal permeability, hydrogen breath test]. Revue Med Vet. 2000;151(7):559-63.

29. Ruaux CG. Cobalamin in companion animals: diagnostic marker, deficiency states and therapeutic implications. Vet J. 2013;196(2):145-52.

30. Sun Y, Sun M, Liu B, Du Y, Rong S, Xu G, Snetselaar LG, Bao W. Inverse association between serum vitamin B12 concentration and obesity among adults in the United States. Front Endocrinol. 2019. https://doi.org/10.3389/fendo.2019.00414.

31. Daviddi G, Ricci MA, De Vuono S, Gentili A, Boni M, Lupattelli G. Folate and vitamin $B 12$ in morbid obesity : the influence of folate on anti-atherogenic lipid profile. Int J Vitam Nutr Res. 2019;4:1-7.
32. Roust LR, DiBaise JK. Nutrient deficiencies prior to bariatric surgery. Curr Opin Clin Nutr Metab Care. 2017;20(2):138-44.

33. Thomas-Valdes S, Tostes MDGV, Anunciacao PC, de Silva BP, Sant'Ana HMP. Association between vitamin deficiency and metabolic disorders related to obesity. Crit Rev Food Sci Nutr. 2017;57(15):3332-43.

34. Ravussin Y, Koren O, Spor A, Leduc C, Gutman R, Stombaugh J, Knight R, Ley RE, Leibel RL. Responses of gut microbiota to diet composition and weight loss in lean and obese mice. Obesity. 2012;20(4):738-47.

35. Turnbaugh PJ, Bäckhed F, Fulton L, Gordon Jl. Diet-induced obesity is linked to marked but reversible alterations in the mouse distal gut microbiome. Cell Host Microbe. 2008:3(4):213-23.

36. Turnbaugh PJ, Ridaura VK, Faith JJ, Rey FE, Knight R, Gordon JI. The effect of diet on the human gut microbiome: a metagenomic analysis in humanized gnotobiotic mice. Sci Transl Med. 2009;1 (6):6ra14.

37. Turnbaugh PJ, Hamady M, Yatsunenko T, Cantarel BL, Duncan A, Ley RE, Sogin ML, Jones WJ, Roe BA, Affourtit JP, et al. A core gut microbiome in obese and lean twins. Nature. 2009;457:480-4.

38. Murphy EF, Cotter PD, Healy S, Marques TM, Sullivan O, Fouhy F, Clarke SF, Toole PW, Quigley EM, Stanton C, et al. Composition and energy harvesting capacity of the gut microbiota: relationship to diet, obesity and time in mouse models. Gut. 2010;59(12):1635-42.

39. McCormack UM. In: Gardiner G, Lawlor PG, editors. Investigation and subsequent manipulation of the intestinal microbiota of pigs, with a view to optimizing feed efficiency. PhD Thesis; 2017.

40. Ferrario C, Taverniti V, Milani C, Fiore W, Laureati M, De Noni I, Stuknyte M, Chouaia B, Riso P, Guglielmetti S. Modulation of fecal Clostridiales Bacteria and butyrate by probiotic intervention with Lactobacillus paracasei DG varies among healthy adults. J Nutr. 2014;144(11):1787-96.

41. Zhang $H$, Dibaise JK, Zuccolo A, Kudrna D, Braidotti M, Yu Y, Parameswaran P, Crowell MD, Wing R, Rittmann BE, et al. Human gut microbiota in obesity and after gastric bypass. Proc Natl Acad Sci U S A. 2009;106(7):2365-70

42. Hill SA, Cave NJ, Forsyth S. Effect of age, sex and body weight on the serum concentrations of cobalamin and folate in cats consuming a consistent diet. J Feline Med Surg. 2018;20(2):135-41.

43. Pinhas-Hamiel O, Doron-Panush N, Reichman B, Nitzan-Kaluski D, Shalitin S, Geva-Lerner L. Obese children and adolescents: a risk group for low vitamin B12 concentration. Arch Pediatr Adolesc Med. 2006;160(9):933-6.

44. Yakut M, Ustun Y, Kabacam G, Soykan I. Serum vitamin B12 and folate status in patients with inflammatory bowel diseases. Eur J Intern Med. 2010;21:320-3.

45. Weese JS, Jalali M. Evaluation of the impact of refrigeration on next generation sequencing-based assessment of the canine and feline fecal microbiota. BMC Vet Res. 2014;10:230.

46. Tal M, Verbrugghe A, Gomez D, Chau C, Weese JS. The effect of storage at ambient temperature on the feline fecal microbiota. BMC Vet Res. 2017; 13(1):1-8.

47. Inness VL, McCartney AL, Khoo C, Gross KL, Gibson GR. Molecular characterisation of the gut microflora of healthy and inflammatory bowel disease cats using fluorescence in situ hybridisation with special reference to Desulfovibrio spp. J Anim Physiol Anim Nutr (Berl). 2007; 91(1-2):48-53.

48. Abecia L, Hoyles L, Khoo C, Frantz N, McCartney AL. Effects of a novel galactooligosaccharide on the faecal microbiota of healthy and inflammatory bowel disease cats during a randomized, double-blind, crossover feeding study. Int J Probiotics Prebiotics. 2010;5(2):61-8.

49. Laflamme DP. Development and validation of a body condition score system for cats: a clinical tool. Feline Pract. 1997;25(5-6):13-8.

50. Baldwin K, Bartges J, Buffington T, Freeman LM, Grabow M, Legred J, Ostwald DJ. AAHA nutritional assessment guidelines for dogs and cats. J Am Anim Hosp Assoc. 2010:46(4):285-96.

51. National Research Council (NRC). Energy. In: Nutrient Requirements of Dogs and Cats. Washington: The National Academies Press; 2006.

52. Michel KE, Anderson W, Cupp C, Laflamme DP. Correlation of a feline muscle mass score with body composition determined by dual-energy $X$-ray absorptiometry. Br J Nutr. 2011;106(S1):S57-9.

53. Nelson RW, Himsel CA, Feldman EC, Bottoms GD. Glucose tolerance and insulin response in normal-weight and obese cats. Am J Vet Res. 1990;51(9):1357-62.

54. Verbrugghe A, Hesta M, Daminet S, Polis I, Holst JJ, Buyse J, Wuyts B, Janssens GP. Propionate absorbed from the colon acts as gluconeogenic substrate in a strict carnivore, the domestic cat (Felis catus). J Anim Physiol Anim Nutr (Berl). 2012;96(6):1054-64. 
55. Brooks D, Churchill J, Fein K, Linder D, Michel K, Tudor K, Ward E, Witzel A. 2014 AAHA weight management guidelines for dogs and cats. J Am Anim Hosp Assoc. 2014;50(1):1-11.

56. Klindworth A, Pruesse E, Schweer T, Peplies J, Quast C, Horn M, Glockner FO Evaluation of general 165 ribosomal RNA gene PCR primers for classical and next-generation sequencing-based diversity syudies. Nucleic Acids Res. 2013. https://doi.org/10.1093/nar/gks808.

57. Sturgeon A. In: Weese JS, editor. Analysis of the Oral and fecal microbiota of companion animals using next-generation sequencing of the $16 \mathrm{~S}$ rRNA gene. MSc Thesis; 2014.

58. Schloss PD, Westcott SL, Ryabin T, Hall JR, Hartmann M, Hollister EB, Lesniewski RA, Oakley BB, Parks DH, Robinson CJ, et al. Introducing mothur: open-source, platform-independent, community-supported software for describing and comparing microbial communities. Appl Environ Microbiol. 2009:75(23):7537-41.

59. Kozich JJ, Westcott SL, Baxter NT, Highlander SK, Schloss PD. Development of a dual- index sequencing strategy and Curation pipeline for analyzing amplicon sequence data on the MiSeq Illumina sequencing platform. Appl Environ Microbiol. 2013;79(17):5112-20.

60. Quast C, Pruesse E, Yilmaz P, Gerken J, Schweer T, Yarza P, Peplies J, Glockner FO. The SILVA ribosomal RNA gene database project: improved data processing and web-based tools. Nucleic Acids Res. 2013;41(D1):D590-6.

61. Edgar RC, Haas BJ, Clemente JC, Quince C, Knight R. UCHIME improves sensitivity and speed of chimera detection. Bioinformatics. 2011:27(16):2194-200.

62. Cole JR, Wang Q, Fish JA, Chai BL, McGarrell DM, Sun YN, Brown CT, PorrasAlfaro A, Kuske CR, Tiedje JM. Ribosomal database project: data and tools for high throughput rRNA analysis. Nucleic Acids Res. 2014;42(D1):D633-42.

63. Gihring T, Green S, Schadt CW. Massively parallel rRNA gene sequencing exacerbates the potential for biased community diversity comparisons due to variable library sizes. Environ Microbiol. 2011;14(2):285-90.

64. Shannon CE. A mathematical theory of communication. Bell Syst Tech J. 1948;27(3):379-423.

65. Simpson E. Measurement of Diversity. Nature. 1949;163:688.

66. Chao A. Nonparametric estimation of the number of classes in a population. Scand J Stat. 1984;11(4):265-70

67. Benjamini Y, Hochberg Y. Controlling the false discovery rate: a practical and powerful approach to multiple testing. J R Stat Soc Series B Stat Methodol. 1995;57(1):289-300.

68. Smith E. Nonparametric estimation of species richness. Biometrics. 1984; 40(1):119-29.

69. Yue J, Clayton M. A similarity measure based on species proportions. Commun Stat Theory Methods. 2005;34(11):2123-31.

70. Lozupone C, Hamady M, Knight R. UniFrac - an online tool for comparing microbial community diversity in a phylogenetic context. BMC Bioinformatics. 2006;7:371.

71. Segata N, Izard J, Waldron L, Gevers D, Miropolsky L, Garrett WS, Huttenhower C. Metagenomic biomarker discovery and explanation. Genome Biol. 2011;12(6):R60.

\section{Publisher's Note}

Springer Nature remains neutral with regard to jurisdictional claims in published maps and institutional affiliations.

Ready to submit your research? Choose BMC and benefit from:

- fast, convenient online submission

- thorough peer review by experienced researchers in your field

- rapid publication on acceptance

- support for research data, including large and complex data types

- gold Open Access which fosters wider collaboration and increased citations

- maximum visibility for your research: over $100 \mathrm{M}$ website views per year

At $\mathrm{BMC}$, research is always in progress.

Learn more biomedcentral.com/submissions 